\title{
Systematization of the Incisions in Nipple-Sparing Mastectomy
}

\section{Marcela Caetano Cammarota ${ }^{1, *}$, Leonardo David Pires Barcelos ${ }^{2}$, Ronan Caputi Silva Dias ${ }^{3}$, Tristão Maurício de Aquino Filho $^{3}$, Alberto Benedik Neto ${ }^{1}$, Fabrício Tavares Mendonça ${ }^{4}$, and José Carlos Daher ${ }^{5}$}

${ }^{1}$ Active member of the Brazilian Society of Plastic Surgery (SBCP), Plastic surgeon preceptor of the Plastic Surgery Service of the Hospital Daher Lago Sul (HDLS), Brasilia, DF, Brazil

${ }^{2}$ Specialist Member of the SBCP, BellEssence Integrated Medicine, Brasília, DF, Brazil

${ }^{3}$ Aspiring member of the SBCP, Resident physician at the Plastic Surgery Service of the HDLS, Brasília, DF, Brazil

${ }^{4}$ Active Member of the Brazilian Society of Anesthesiology, Anesthesiologist co-responsible for the education center at the Hospital de Base do Distrito Federal, Assistant Anesthesiologist at the Hospital Daher Lago Sul (HDLS), Brasilia, DF, Brazil

${ }^{5}$ Active member of the Brazilian Society of Plastic Surgery (SBCP), Plastic surgeon chair of the Plastic Surgery Service of the Daher Lago Sul Hospital (HDLS), Brasilia, DF, Brazil

* Corresponding author: Marcela Caetano Cammarota, Active member of the Brazilian Society of Plastic Surgery (SBCP), Plastic surgeon preceptor of the Plastic Surgery Service of the Hospital Daher Lago Sul (HDLS), Brasilia, DF, Brazil, Tel: +55(61)3425-3340; E-mail: marcelacammarota@yahoo.com.br

Received: 19 Apr, 2021 | Accepted: 23 Jun, 2021 | Published: 22 Jul, 2021

Citation: Cammarota MC, Barcelos LDP, Dias RCS, de Aquino Filho TM, Neto AB, et al. (2021) Systematization of the Incisions in NippleSparing Mastectomy. J Surg Open Access 7(4): dx.doi.org/10.16966/2470-0991.245

Copyright: (c) 2021 Cammarota MC, et al. This is an open-access article distributed under the terms of the Creative Commons Attribution License, which permits unrestricted use, distribution, and reproduction in any medium, provided the original author and source are credited.

\begin{abstract}
Introduction: Nipple-Sparing Mastectomy (NSM) is a consolidated technique that has been used for years with good aesthetic results. Its indication is usually limited by breast size, due to difficulty repositioning the Nipple-Areola Complex (NAC) and treating excess skin in large breasts. The challenge in these cases is to maximize the aesthetic result without increasing the risk of necrosis and other complications.

General objective: Describe a systematization of incisions according to breast size and NAC position and compare the impact on the outcome.

Method: Evaluation of patients submitted to mastectomies with NSM and Direct-to-Implant (DTI) reconstruction. The breasts were stratified into 3 groups according to the size and in 4 groups according to the position of the NAC (Regnault classification). Six different types of incisions were studied.

Results: The total sample included 157 breasts. The most-frequently performed incisions were the horizontal periareolar sickle incision with $48.4 \%$, followed by the oblique periareolar sickle incision with triangular resection with $15.9 \%$. The most common complication was excess skin in $26.1 \%$. The minimum follow-up time was 1 year.

Conclusions: In this sample, we found that the horizontal periareolar sickle incision could be widely used in medium-sized breasts. Breasts larger than 400grams tended to have worse outcomes. The use of incisions that allowed for the removal of skin produced the best results in large breasts. This study facilitated the understanding of the dynamics of the incisions and guided the type of incision according to breast size and position of the NAC.
\end{abstract}

Keywords: Breast neoplasms; Breast; Mastectomy; Extended radical mastectomy; Immediate reconstruction; Implant reconstruction; Modified radical mastectomy; Breast implant

\section{Introduction}

In the last two decades, we have seen drastic changes in the treatment of breast cancer. Our advancing understanding of the biology of tumors and of cytogenetics has made treatment more specific and effective, so that the operations have become less mutilating and oncologically safer [1]. In addition to these changes, we also see that the incidence of these tumors today occurs in increasingly younger women, thus changing the breast reconstruction profile [2].

The Skin-Sparing Mastectomy (SSM) with immediate reconstruction was first reported by Freeman in 1962 [3]. Since then, the challenge has been to offer reconstructions to women that did not require flaps, leaving fewer scars. The implant manufacturing industry today offers a wide variety of shapes and sizes, and increased safety and durability [4].

Throughout the world, immediate reconstructions with expander implants (in two stages - expander/implant) and direct reconstructions with implant placement (Direct-to-Implant (DTI)) have become the most frequently recommended interventions because they associate safety and rapid recovery with excellent aesthetic results [5]. The greatest challenge of this type of approach is its applicability in large breasts, where the excess skin, resulting from the adenomastectomy, will inevitably require correction [6]. In total mastectomies with removal of the Nipple-Areola-Complex (NAC), the adjustment of the skin can be performed immediately and the type of incision can be quite varied - without offering greater risks and left to the discretion of the surgical team and their expertise. 
The Nipple-Sparing Mastectomy (NSM) has had a profound impact on the potential of the aesthetic results of reconstructions [7]. Recently, the broadening of the indications for this NSM technique and the increase of risk-reducing mastectomies, have had a positive impact on the acceptance and satisfaction of patients with the results, as well as an equivalence in the oncologic monitoring, when compared to skin-sparing mastectomies and total mastectomies [1,8-11].

However, the recommendation of NSM has been limited by breast size and ptosis, due to the difficulty in repositioning the NAC and handling the excess skin in large breasts, which can increase the chance of compromising the flap and losing the NAC [12].

The average incidence of NAC necrosis after mastectomy is 7\% [13]. Careful preoperative planning can facilitate access and the removal of skin over the tumor region without affecting the result and can sometimes decrease the chance of vascular damage. In addition, some incisions allow for a reduction of skin redundancy as early as in the first stage of surgery [14]. There is still poor data regarding how the type of incision affects results [14].

\section{Objective}

Compare the incisions on nipple sparing mastectomies and suggest a systematization for the planning of the incisions in accordance with breast size and NAC position.

\section{Method}

A retrospective medical record review was conducted, encompassing patients undergoing immediate breast reconstruction surgery using the DTI technique. All patients operated on by the same surgeon between May 2016 and May 2020 was analyzed.

The inclusion criteria were patients with breast cancer who underwent total uni or bilateral mastectomies with preservation of the NAC, even when unilateral, and immediate breast reconstruction using the DTI technique. All patients provided their individual consent for the use of clinical photographs and information for publication in this manuscript. The exclusion criteria were patients who had medical records with incomplete data or who had not concluded their breast reconstruction in the period under study. Ethics approval was sought but not required as the technique has been previously reported.

\section{Surgical technique}

All cases were performed following the same surgical technique standardization:

- Incision planning in agreement with the mastology team.

- The implant size is chosen in accordance with patient's desire. It is placed completely in a submuscular pocket.

- Drain placement and adjustment of excess skin using the previously planned incisions.

- Textured silicone prostheses with anatomical or round shape with high projection were used.

The demographic data studied were age, laterality, comorbidities, smoking, BMI (Body Mass Index), treatment with neoadjuvant or adjuvant chemotherapy or radiotherapy, number of surgeries performed per patient and follow-up time.

The patients were divided into 3 groups according to breast size, which was based on the weight of the surgical piece and classified as: Small-S patients (whose breasts weighed less than 250grams), Median-M patients (whose breasts weighed between 250 and 399grams) and Large-L patients (whose breast weighed more than 400grams).

The position of the NAC was classified according to the Regnault classification as: Type 1 Normal: Nipple above the level of the inframammary fold.

Type 2 Minor ptosis: Nipple at the level of the inframammary fold. fold.

Type 3 Moderate ptosis: Nipple below the level of the inframammary

Type 4 Severe ptosis: Nipple below the level of the inframammary fold, at the lower contour of the gland [15].

The incisions were classified as: Radial incision- Type 1 , Inframammary fold incision-Type 2, Horizontal periareolar sickle incision-Type 3, Oblique periareolar sickle incision with triangular resection-Type 4, Oblique periareolar sickle incision with sickle resection-Type 5 and Vertical periareolar sickle incision-Type 6, used in patients with previous mammoplasty (Figure 1).

Subgroup classification was also performed, associating breast size $(\mathrm{S}, \mathrm{M}, \mathrm{L})$ with the position of the NAC $(1,2,3,4)$ in S1, S2, S3, S4; M1, M2, M3, M4; L1, L2, L3, L4 (Figure 2).

Post-operative complications of interest were hematoma, surgical site infection, partial or total thickness NAC necrosis, excess skin after the first operation, adhesions or unsightly scar, capsular contracture, and change in implant position (rotation or height change in relation to the mammary fold). In this series, we did not have any loss of implants.

The results were classified by two external plastic surgeons as excellent, good, fair and poor, who reviewed the photos after first stage operation. Excellent and good results were grouped as satisfactory and fair and poor results, as unsatisfactory.

\section{Statistical analysis}

The frequency of the categorical data was compared by Fisher's exact test or the chi-square test $\left(\mathrm{X}^{2}\right)$, as appropriate. The categorical variables were expressed as absolute numbers and percentages. Results were considered statistically significant at $\mathrm{p}<.05$. The statistical analyses were performed using SPSS for Macintosh (Statistical Package for the Social Sciences, Chicago, IL, USA) version 20.0.

\section{Results}

Overall, 107 patients were evaluated with a mean age of 48 years. $47 \%$ of these patients were bilateral, totaling 157 breasts submitted to NSM and DTI.

The mean BMI was $25.52 \mathrm{~kg} / \mathrm{m}^{2}$. The mean breast weight was $346 \mathrm{~g}$ $(142 \mathrm{~g}$ to $697 \mathrm{~g})$ and the average size of implants was $390 \mathrm{ml}(275 \mathrm{ml}$ to $555 \mathrm{ml})$.

The number of operations performed in patients who completed the reconstruction, ranged from 1 to 4 (mean 1.56) and the minimum follow-up time was 1 year.

The most frequently performed incision was Type 3 (horizontal periareolar sickle incision) in 76 breasts $(48.4 \%)$, followed by the Type 4 (oblique periareolar sickle incision with triangular resection) in 25 breasts (15.9\%). The least used was the radial incision (Type 1), performed in only 4 breasts (2.5\%) (Figure 3 ).

Partial NAC necrosis was observed in $16.6 \%$ of cases, with resolution after the use of vasodilators and dressings. No total necrosis of NAC was observed in any patient and mild cases of spontaneously resolving epidermolysis were not considered 


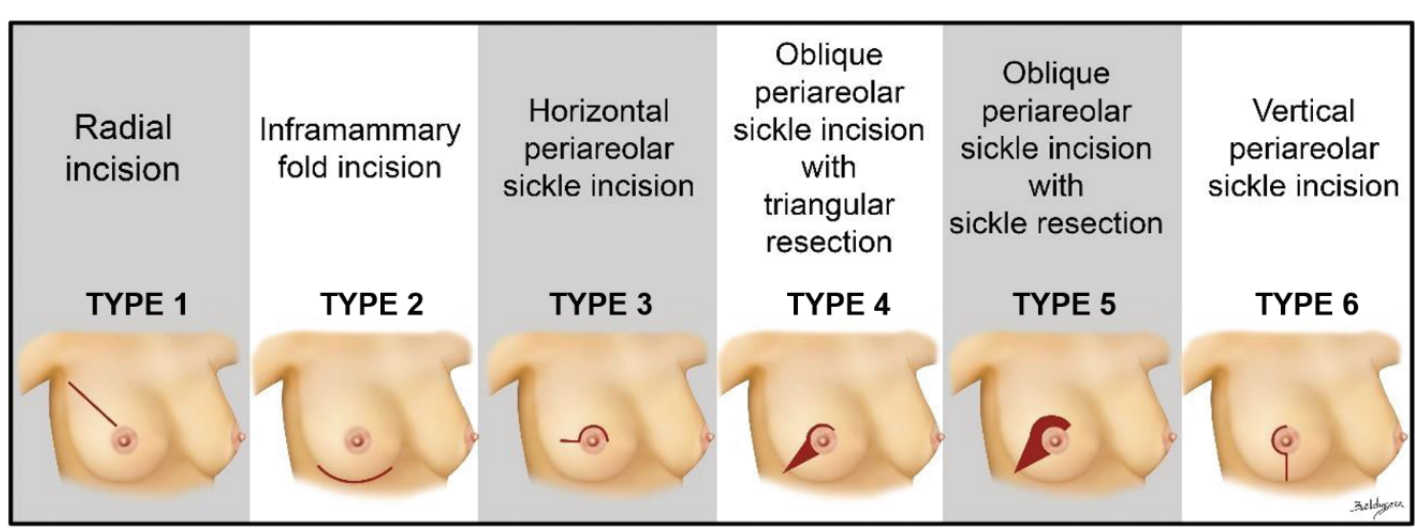

Figure 1: Classification of the incisions.

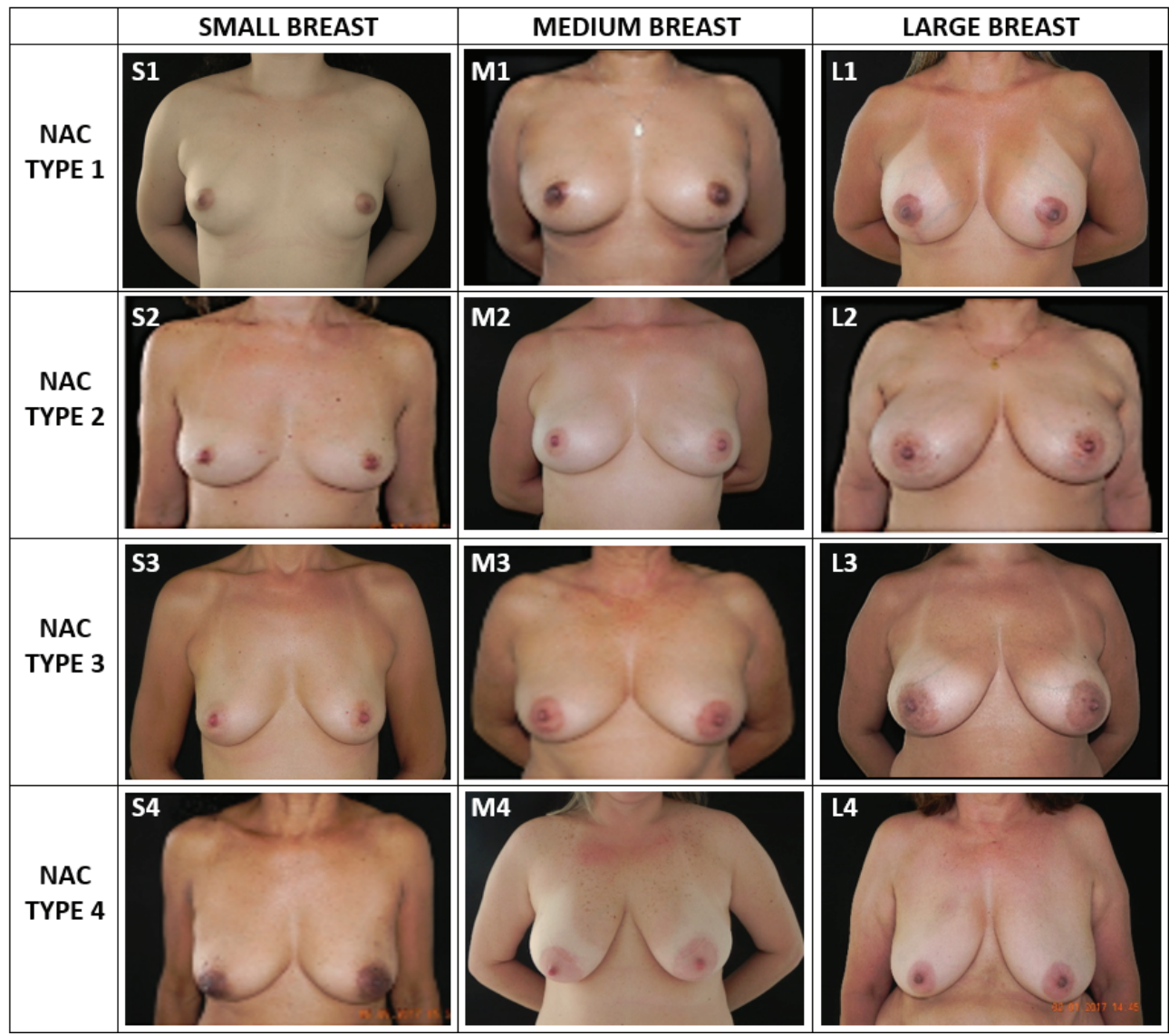

Figure 2: Subgroup classification: S1, S2, S3, S4; M1, M2, M3, M4; L1, L2, L3, L4.

complications. The most common complication was excess skin after the first operation in $26.1 \%$ of cases, until other types of large breast incisions were used to avoid this outcome. We observed capsular contracture in $10.8 \%$ of cases, $6.4 \%$ with adhesions, hematoma in $4.5 \%$, unsightly scar in $3.8 \%$, infection in $3.8 \%$ and altered implant position in $1.9 \%$ of cases.

As shown in table 1, regarding breast size, larger breasts tended to have the worst results. Breast sizes $\mathrm{S}$ and $\mathrm{M}$ had the best results $(\mathrm{p}<.001)$.
Regarding NAC position (Regnault classification), the results showed that Type 1 had $100 \%$ of satisfactory results. The worst results occurred in the breasts with higher degrees of ptosis $(\mathrm{p}=.021)$ (Table 2).

By further subdividing the groups according to breast size and position of the NAC, we find that some subgroups only had satisfactory results (excellent or good): S1, S2, S4, M1 and L1 (p<.013), as patients with Type 1 NAC have been previously demonstrated to have better results (Table 3 ). 


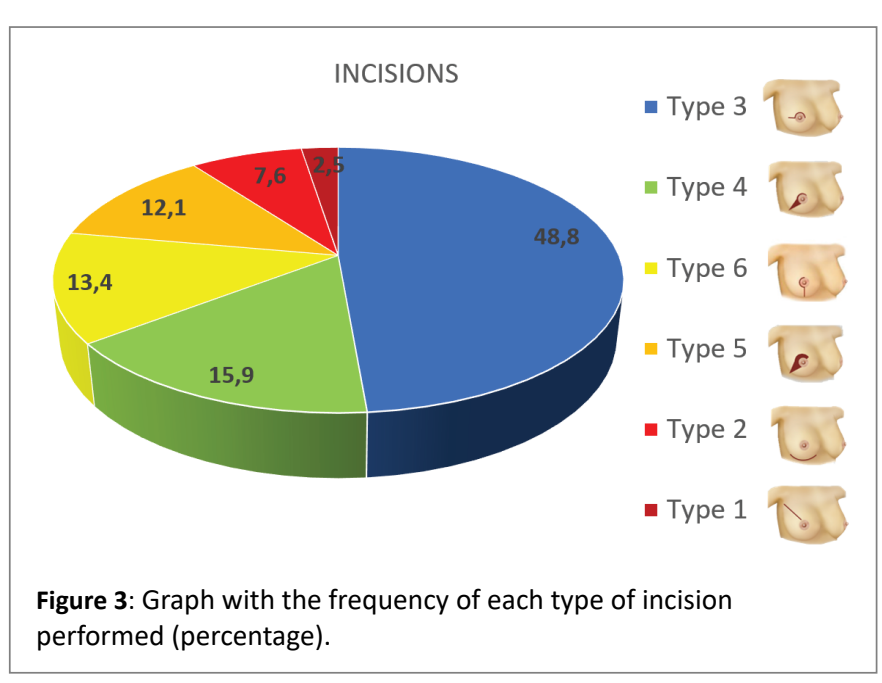

Table 1: Breast size $\times$ satisfactory result $(p<.001)(\mathrm{S}$ : Small; M: medium; L: Large).

\begin{tabular}{|c|c|c|c|c|c|}
\hline \multirow{2}{*}{ Size } & \multicolumn{2}{|c|}{ Satisfactory } & \multicolumn{2}{c|}{ Unsatisfactory } & \\
\cline { 2 - 6 } & Excellent & Good & Fair & Poor & Total \\
\hline S & $25(89.3 \%)$ & $1(3.6 \%)$ & $2(7.1 \%)$ & $0(0.0 \%)$ & $28(17.8 \%)$ \\
\hline M & $47(70.1 \%)$ & $15(22.4 \%)$ & $3(4.5 \%)$ & $2(3.0 \%)$ & $67(42.7 \%)$ \\
\hline L & $29(46.8 \%)$ & $17(27.4 \%)$ & $13(21.0 \%)$ & $3(4.8 \%)$ & $62(39.5 \%)$ \\
\hline & $101(64.3 \%)$ & $33(21.0 \%)$ & $18(11.5 \%)$ & $5(3.2 \%)$ & 157 \\
\hline
\end{tabular}

(Excellent and Good=Satisfactory/Fair and Poor=Unsatisfactory)

Table 2: NAC position $\times$ satisfactory result $(p=.021)$.

\begin{tabular}{|l|c|c|c|c|c|}
\hline & \multicolumn{2}{|c|}{ Satisfactory } & \multicolumn{2}{c|}{ Unsatisfactory } & \\
\hline & Excellent & Good & Fair & Poor & Total \\
\hline NAC 1 & $33(86.8 \%)$ & $5(13.2 \%)$ & $0(0.0 \%)$ & $0(0.0 \%)$ & $38(24.2 \%)$ \\
\hline NAC 2 & $27(61.4 \%)$ & $10(22.7 \%)$ & $5(11.4 \%)$ & $2(4.5 \%)$ & $44(28 \%)$ \\
\hline NAC 3 & $30(60.0 \%)$ & $11(22.0 \%)$ & $7(14.0 \%)$ & $2(4.0 \%)$ & $50(31.9 \%)$ \\
\hline NAC 4 & $11(44.0 \%)$ & $7(28.0 \%)$ & $6(24.0 \%)$ & $1(4.0 \%)$ & $25(15.9 \%)$ \\
\hline & $101(64.3 \%)$ & $33(21.0 \%)$ & $18(11.5 \%)$ & $5(3.2 \%)$ & 157 \\
\hline
\end{tabular}

(NAC: Regnault classification)

(Excellent and Good=Satisfactory/Fair and Poor=Unsatisfactory)

For the subgroups with unsatisfactory results (fair or poor) we studied the types of incision and their statistical significance (Table 4). We verified that

- The M2 breast subgroup had better results when type 3 incision (horizontal periareolar sickle incision) were made ( $\mathrm{p}=.02)$.

- The L3 breast subgroup had better result when subjected to surgical incisions of types 4 and 5. For these breasts, we verified a statistical tendency for worse results when type 3 incisions were made $(\mathrm{p}=.018)$.

- Subgroup L4 had better results with type 5 incisions and worse results with types 3 and 4 incisions $(\mathrm{p}<.001)$.

When analyzing the presence of complications in relation to breast size, a significant result was found for large breasts $(\mathrm{L})(\mathrm{p}=.013)$ (Table
Table 3: Subgroup according to breast size and position of the NAC $x$ results $(p<.013)$.

\begin{tabular}{|l|c|c|c|c|}
\hline & \multicolumn{2}{|c|}{ Satisfactory } & \multicolumn{2}{c|}{ Unsatisfactory } \\
\hline & Excellent & Good & Fair & Poor \\
\hline S1 & $7(87.5 \%)$ & $1(12.5 \%)$ & 0 & 0 \\
\hline S2 & $9(100.0 \%)$ & 0 & 0 & 0 \\
\hline S3 & $5(71.4 \%)$ & 0 & $2(28.6 \%)$ & 0 \\
\hline S4 & $4(100.0 \%)$ & 0 & 0 & 0 \\
\hline M1 & $19(90.5 \%)$ & $2(9.5 \%)$ & 0 & 0 \\
\hline M2 & $13(56.5 \%)$ & $7(30.4 \%)$ & $1(4.3 \%)$ & $2(8.7 \%)$ \\
\hline M3 & $11(61.1 \%)$ & $6(33.3 \%)$ & $1(5.6 \%)$ & 0 \\
\hline M4 & $4(80.0 \%)$ & 0 & $1(20.0 \%)$ & 0 \\
\hline L1 & $7(77.8 \%)$ & $2(22.2 \%)$ & 0 & 0 \\
\hline L2 & $5(41.7 \%)$ & $3(25.0 \%)$ & $4(33.3 \%)$ & 0 \\
\hline L3 & $14(56.0 \%)$ & $5(20.0 \%)$ & $4(16.0 \%)$ & $2(8.0 \%)$ \\
\hline L4 & $3(18.8 \%)$ & $7(43.8 \%)$ & $5(31.3 \%)$ & $1(6.3 \%)$ \\
\hline
\end{tabular}

5). Table 6 show the distribution of the complication excess skin in different breast sizes: it resulted to be associated with large breasts $(\mathrm{p}=.033)$.

\section{Discussion}

NSM can preserve the skin envelope of the breast almost in its entirety, including the nipple-areola complex [16]. Despite the improvements in the aesthetic results shown by studies using this technique, as well as safety regarding the oncologic results, NSM remains in constant evolution [17].

The average age of our sample was 48 years, which is low for breast cancer statistics. As well as our, other studies have shown that patients undergoing NSM tend to be younger $[18,19]$.

The average body mass index of our sample was $24.83 \mathrm{~kg} / \mathrm{m}^{2}$. Davies identified that a high body mass index, breast resections greater than $750 \mathrm{~g}$ and long distances from NAC to the sternal furcula are factors associated with a higher incidence of skin flap necrosis, [20] while Munhoz identified a high body mass index, breast weight and hemiperiareolar and wise-type incisions as factors associated with higher incidences of complications [21-24].

We used radial and inframammary fold incisions based on literature data describing them as the most used for NSM, with lower necrosis rate [16]. However, the occurrence of flap necrosis was greater in this series, and those incisions turn out to have exceptional indication. The radial incision proved to be useful but restricted to cases of NAC type 1 , because it does not allow for the superior repositioning of NAC.

When we started breast reconstruction with DTI, the incision of choice was Horizontal sickle incision (Type 3). It was the most prevalent incision, performed in 76 breasts (48.4\%) and it is undoubtedly the most used in the world. It proved to be effective in vascular preservation, in addition to allowing for the repositioning of the NAC in cases of ptosis or lateralization [16].

As the technique evolved to perform DTI on larger breasts, a second surgical intervention was necessary, adding further scars to the breast. Excess scars, however, are not well accepted by patients (Figure 4 ). 

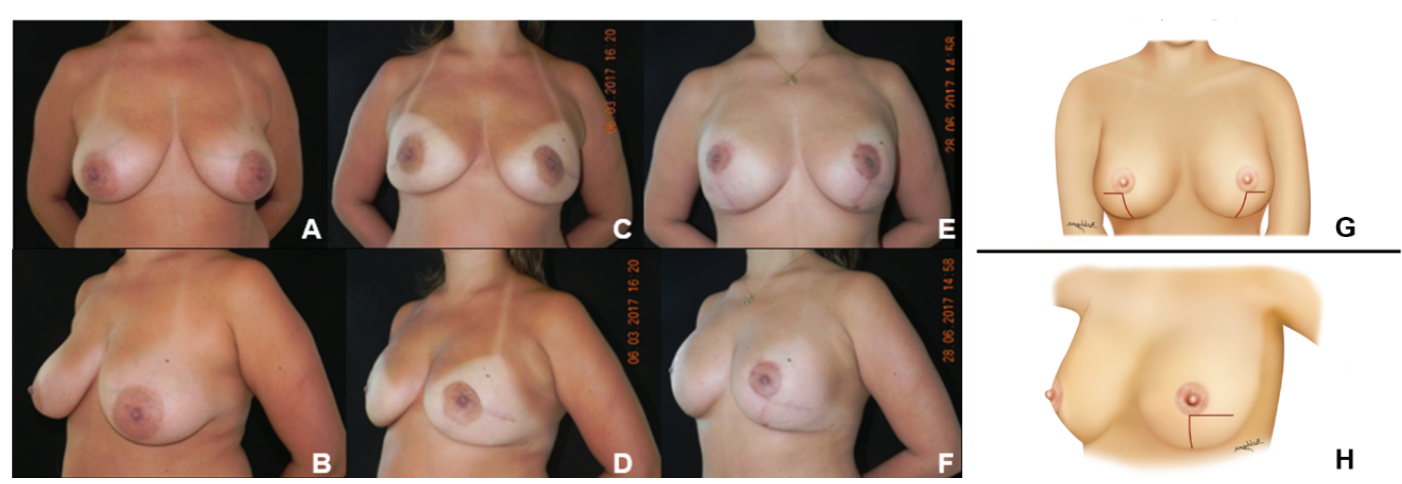

Figure 4: Bilateral NSM with horizontal sickle incision and DTI reconstruction. (A, B): Pre-operative; (C, D): Post-operative

$1^{\text {st }}$ intervention with excess skin; (E, F): Post-operative.

$2^{\text {nd }}$ intervention with removal of excess skin in inverted $T ;(G, H)$ : Diagram showing the incisions resulting from 2 procedures.

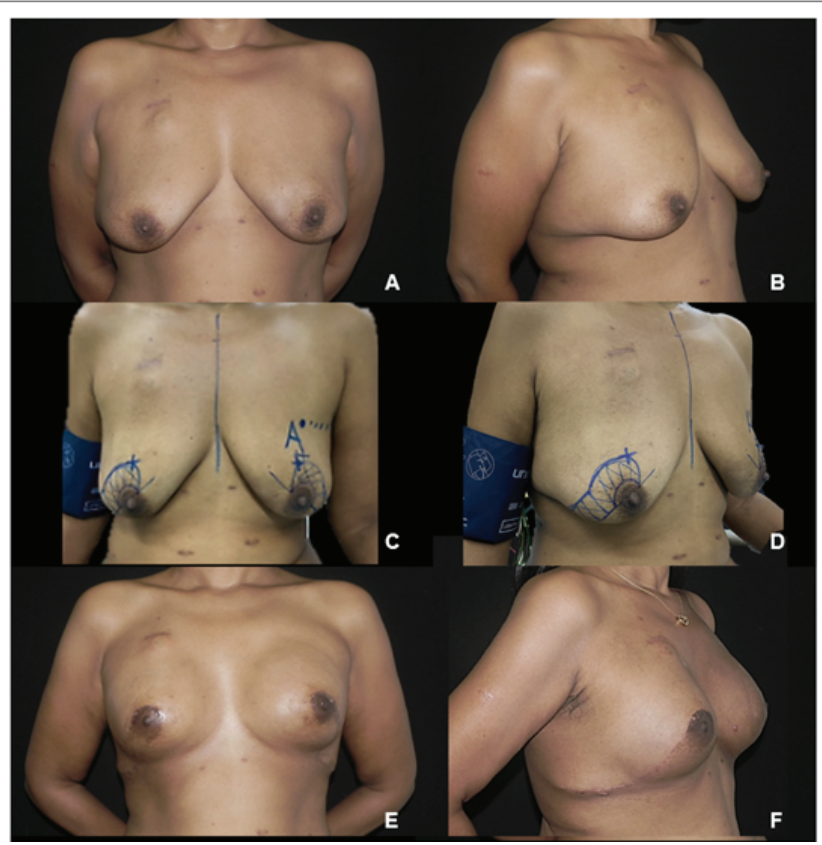

Figure 5: Bilateral NSM with incision and resection in oblique sickle and DTI reconstruction. (A, B): Pre-operative; (C, D): surgical marking of skin resection; $(E, F)$ : Post-operative period of 6 months.

The likelihood of NAC malposition has led many surgeons to consider large breasts as a contraindication in DTI reconstructions. Technical modifications to the conventional NAC-sparing procedure were therefore developed, addressing large or ptotic breasts with the purpose of maintaining a viable NAC, properly positioned on the top of the breast mound [24].

Some well-established techniques to skin reduction mastectomy are described in literature. Wise pattern incision and vertical mastopexy incision was widely used in a systematic revision [23].

Folli S, et al. shows that wise incision is a good technique for correct large and ptotic breasts using modified McKissock's vertical bi-pedicle dermal flap and helps to maintain the viability of NAC.

However, nipple viability is critical; necrosis is reported to occur in less than $10 \%$ of the cases in some series but ranges from $0 \%$ to $48 \%$ in the literature. Reduced skin perfusion in these techniques explains the higher complication rates due to flap necrosis. Careful dissection to avoid excessively thin mastectomy flaps its desirable, although in cancer patients thick flaps are to be avoided As well as glandular remnants [22].

Some authors suggest a breast reduction before mastectomy to resolve this problem $[6,12]$. In our series, 7 patients (10 breasts $10 \%)$ underwent previous cosmetic mammoplasty, 2 of whom had an incidental diagnosis of cancer and performed bilateral mastectomy 60 days after cosmetic surgery. We believe that such procedure should only be applied to patients undergoing risk-reducing mastectomies, since aesthetic objectives should not be given priority to, in relation to cancer treatments.

Based on these observations and on the data mentioned in table 6 , we strategically altered the incision to allow for the repositioning of the $\mathrm{NAC}$ and the treatment of excess skin in the first surgical intervention, 
Table 4: Incision type $\times$ Result by subgroup.

\begin{tabular}{|c|c|c|c|}
\hline S1 & Satisfactory & Unsatisfactory & $P$ value \\
\hline Incision 3 & 8 & 0 & - \\
\hline S2 & Satisfactory & Unsatisfactory & $P$ value \\
\hline Incision 1 & 1 & 0 & \multirow{3}{*}{0,573} \\
\hline Incision 3 & 7 & 0 & \\
\hline Incision 4 & 1 & 0 & \\
\hline S3 & Satisfactory & Unsatisfactory & $P$ value \\
\hline Incision 1 & 1 & 0 & \multirow{2}{*}{0,608} \\
\hline Incision 3 & 4 & 2 & \\
\hline S4 & Satisfactory & Unsatisfactory & $P$ value \\
\hline Incision 3 & 4 & 0 & - \\
\hline M1 & Satisfactory & Unsatisfactory & $P$ value \\
\hline Incision 2 & 4 & 0 & \multirow{3}{*}{0,868} \\
\hline Incision 3 & 10 & 0 & \\
\hline Incision 6 & 7 & 0 & \\
\hline M2 & Satisfactory & Unsatisfactory & $P$ value \\
\hline Incision 2 & 2 & 2 & \multirow{4}{*}{$0,020 *$} \\
\hline Incision 3 & 12 & 0 & \\
\hline Incision 4 & 5 & 1 & \\
\hline Incision 6 & 1 & 0 & \\
\hline M3 & Satisfactory & Unsatisfactory & $P$ value \\
\hline Incision 2 & 4 & 0 & \multirow{5}{*}{0,714} \\
\hline Incision 3 & 5 & 1 & \\
\hline Incision 4 & 3 & 0 & \\
\hline Incision 5 & 4 & 0 & \\
\hline Incision 6 & 1 & 0 & \\
\hline M4 & Satisfactory & Unsatisfactory & $P$ value \\
\hline Incision 3 & 3 & 1 & \multirow{2}{*}{0,401} \\
\hline Incision 5 & 1 & 0 & \\
\hline L1 & Satisfactory & Unsatisfactory & $P$ value \\
\hline Incision 4 & 3 & 0 & \multirow{2}{*}{0,670} \\
\hline Incision 6 & 6 & 0 & \\
\hline L2 & Satisfactory & Unsatisfactory & $P$ value \\
\hline Incision 1 & 1 & 0 & \multirow{4}{*}{0,682} \\
\hline Incision 3 & 2 & 1 & \\
\hline Incision 4 & 3 & 1 & \\
\hline Incision 6 & 2 & 2 & \\
\hline L3 & Satisfactory & Unsatisfactory & $P$ value \\
\hline Incision 1 & 1 & 0 & \multirow{5}{*}{$0,018^{*}$} \\
\hline Incision 3 & 4 & 6 & \\
\hline Incision 4 & 6 & 0 & \\
\hline Incision 5 & 7 & 0 & \\
\hline Incision 6 & 1 & 0 & \\
\hline L4 & Satisfactory & Unsatisfactory & $P$ value \\
\hline Incision 3 & 2 & 4 & \multirow{4}{*}{$0,0000002^{*}$} \\
\hline Incision 4 & 0 & 2 & \\
\hline Incision 5 & 7 & 0 & \\
\hline Incision 6 & 1 & 0 & \\
\hline
\end{tabular}

without increasing the risk of damaging the vascularization. The incisions that achieved this goal were based on studies by Hollander and we called them oblique periareolar sickle incision [24].

The statistical analyses showed that changing from type 3 incision (horizontal sickle) to 4 or 5 (oblique periareolar sickle incision with triangular resection or sickle resection) in the L3 group (large breast with ptosis grade 3), was effective in avoiding excess skin in " $L$ " patients (Table 4 and Figure 5). These incisions ( 4 or 5) produced good aesthetic results after a single procedure, without increasing the rate of complications. However, in cases of unilateral breast reconstruction requiring contra lateral breast reduction, we must consider the symmetry between the scars, which is possible with the use of the vertical incision (type 6) [25]. In order to avoid flap necrosis, incisions 4 and 5 are also well indicated in contra lateral reduction mammoplasty, for symmetrization with similar scars.

For large and ptotic breast, may be through the association of Spira's mastopexy, in which anperiareolar incison is performed for the invagination of breast tissue, thus achieving the correct positioning of the NAC [26].

Frey JD, et al. evaluated 809 mastectomies and found that patients with large breasts had more necrosis of the mastectomy flap and NAC, explants, infection, abscess and seroma [6]. Likewise, Munhoz $\mathrm{AM}$, et al. reported that, in relation to the risk factors regarding this procedure, breast volumes larger than $540 \mathrm{~g}$ had higher probability of developing complications when compared to patients with smaller breasts [21]. Changing incisions for large breasts produced a reduction in complication (Tables 5 and 6), along with the possibility of better repositioning of NAC without increasing the relative risk of damage, as mentioned in the literature. We also observed a reduction in the number of surgical interventions.

The partial necrosis of NAC observed in 26 cases (16.6\%) can be correlated with other findings in the literature. For NAC to remain viable at least 1 perforator of the internal thoracic artery is to be preserved [27]. In total mastectomies, these vessels constitute the main blood supply to the NAC and must be respected.

Based on the data showed above, we suggest a systematization of incision indication according to breast size and NAC position, due to the observation of better results with fewer operative procedures in this series (Figure 6).

Table 5: Breast size $\times$ General complications $(p=.013)$

\begin{tabular}{|l|c|c|c|}
\hline \multirow{2}{*}{ Size } & \multicolumn{3}{|c|}{ General Complications } \\
\cline { 2 - 4 } & N0 & Yes & P value \\
\hline S & $13(46.4 \%)$ & $15(53.6 \%)$ & \\
\hline M & $33(49.3 \%)$ & $34(50.7 \%)$ & \\
\hline L & $19(30.6 \%)$ & $43(69.4 \%)$ & $\mathrm{P}=0,013$ \\
\hline
\end{tabular}

Table 6: Breast size $\times$ Skin excess $(p=.033)$.

\begin{tabular}{|l|c|c|c|}
\hline \multirow{2}{*}{ Size } & \multicolumn{3}{|c|}{ Skin Excess } \\
\cline { 2 - 4 } & No & Yes & P value \\
\hline S & $24(85.7 \%)$ & $4(14.3 \%)$ & \\
\hline M & $53(79.1 \%)$ & $14(20.9 \%)$ & \\
\hline L & $39(62 . \%)$ & $23(37.1 \%)$ & $\mathrm{P}=0,033$ \\
\hline
\end{tabular}




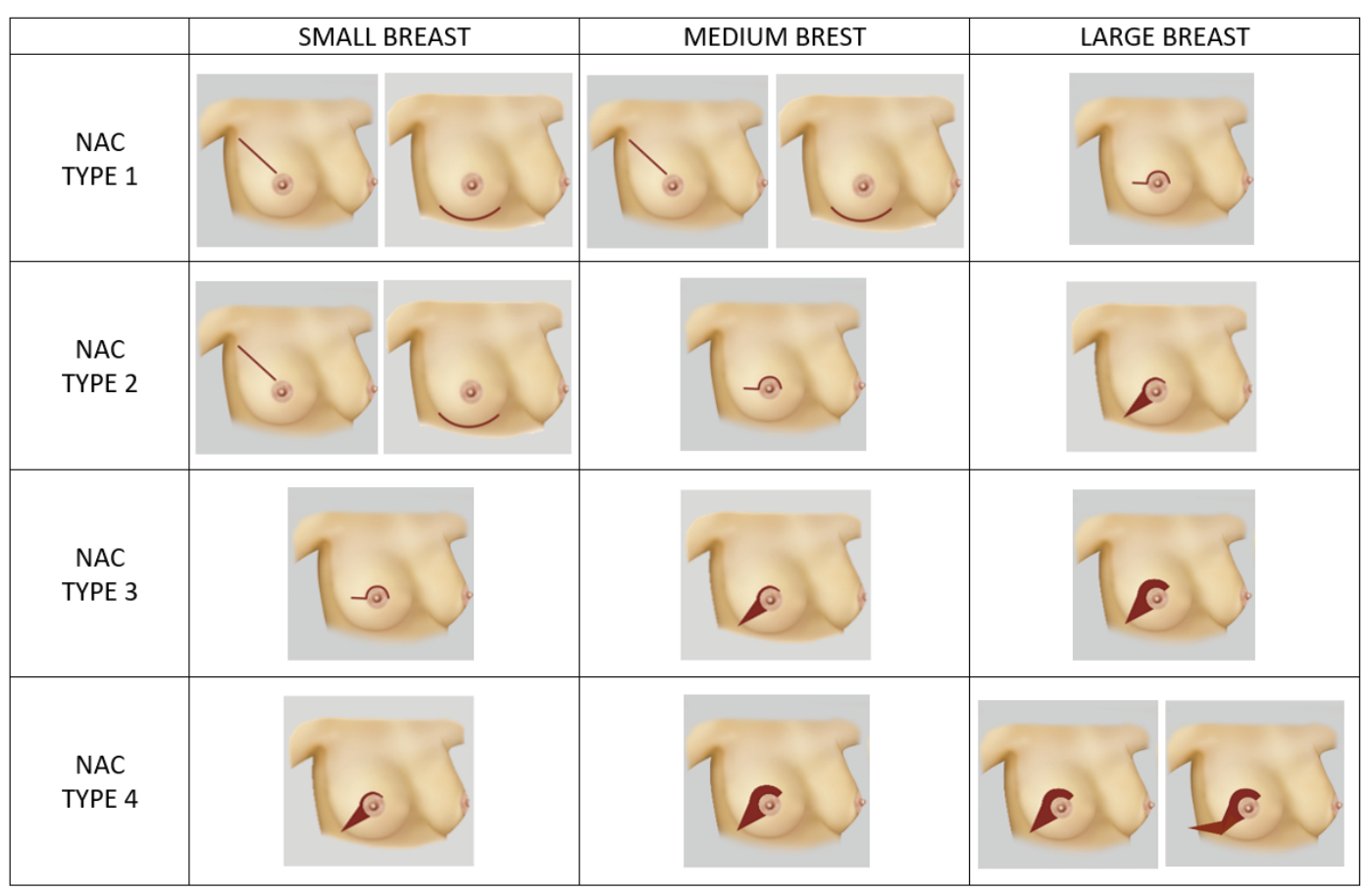

Figure 6: Systematization of incision indication according to breast size and NAC position.

\section{Conclusion}

We conducted an in-depth study of the results of breast reconstructions with DTI and NSM, stratifying the breast groups according to size and positioning of the NAC, and we were able to see that the horizontal sickle incision can be used in most cases and is associated with good results in medium-sized breasts.

Large breasts tend to have worse results with this incision and this fact is correlated with the large excess of skin that remains after the first stage of reconstruction.

The use of incisions that allow skin removal-oblique periareolar sickle incision with triangular resection (incision 4) and oblique periareolar sickle incision with sickle resection (incision 5) produced the best results in large breasts, which enabled the treatment of excess skin, better shaping of the breast and repositioning of the NAC, significantly reducing, or avoiding the second surgical step. These two types of incisions also prevented the addition of scars on the breast in the second surgery, by allowing the same incision to be converted into inverted T-type scars, when necessary.

Thus, we achieved our goal in understanding the dynamics of the incisions: which incision should be recommended for which type of breast and how to obtain the best possible result with fewer complications, even when performing this surgery on large breasts.

\section{Declaration Competing of Interest}

The authors disclose no conflicts of interest related to the current work.

\section{Funding}

There was no funding obtained from any source for the preparation of this manuscript.

\section{References}

1. De La Cruz L, Moody AM, Tappy EE, Blankenship SA, Hecht EM (2015) Overall Survival, Disease-Free Survival, Local Recurrence, and Nipple-Areolar Recurrence in the Setting of Nipple-Sparing Mastectomy: A Meta-Analysis and Systematic Review. Ann Surg Oncol 22: 3241-3249.

2. Cammarota MC, dos Santos GC, Daher JC, Esteves BP, Barcelos LDP, et al. (2018) Breast reconstruction in young women and their peculiarities. Rev Bras Cir Plást 33: 3-11.

3. Toth BA, Lappert P (1991) Modified skin incisions for mastectomy: the need for plastic surgical input in preoperative planning. Plast Reconstr Surg 87: 1048-1053.

4. Gui GP, Tan SM, Faliakou EC, Choy C, A'Hern R, et al. (2003) Immediate breast reconstruction using biodimensional anatomical permanent expander implants: a prospective analysis of outcome and patient satisfaction. Plast Reconstr Surg 111: 125-138.

5. Tostes RO, Andrade Júnior JC, Silva KD, Couto AD, Ribeiro GD, et al. (2005) Immediate breast reconstruction with retromuscular silicone prosthesis: standardization of muscular flaps. Rev Bras Cir Plást 20: 213-219.

6. Frey JD, Salibian AA, Karp NS, Choi M (2018) The Impact of Mastectomy Weight on Reconstructive Trends and Outcomes in Nipple-Sparing Mastectomy: Progressively Greater Complications with Larger Breast Size. Plast Reconstr Surg 141: 795e-803e.

7. Yueh J, Houlihan MJ, Slavin SA, Lee BT, Pories SE, et al. (2009) Nipplesparing mastectomy: evaluation of patient satisfaction, aesthetic results, and sensation. Ann Plast Surg 62: 586-590.

8. Hall-Findlay EJ (2016) Discussion: the blood supply of the breast revisited. Plast Reconstr Surg 137: 1398-1400. 
9. Wellisch DK, Schain WS, Noone RB, Little JW $3^{\text {rd }}$ (1987) The psychological contribution of nipple addition inbreastreconstruction. Plast Reconstr Surg 80: 699-704.

10. Benediktsson KP, Perbeck L (2008) Survival in breast cancer after nipple-sparing subcutaneous mastectomy and immediate reconstruction with implants: a prospective trial with 13 years median follow-up in 216 patients. Eur J Surg Oncol 34: 143-148.

11. Garwood ER, Moore D, Ewing C, Hwang ES, Alvarado M, et al. (2009) Total skin-sparing mastectomy: complications and local recurrence rates in 2 cohorts of patients. Ann Surg 249: 26-32.

12. Spear SL, Rottman SJ, Seiboth LA, Hannan CM (2012) Breast reconstruction using a staged nipple-sparing mastectomy following mastopexy or reduction. Plast Reconstr Surg 129: 572-581.

13. Endara M, Chen D, Verma K, Nahabedian MY, Spear SL (2013) Breast reconstruction following nipple-sparing mastectomy: a systematic review of the literature with pooled analysis. Plast Reconstr Surg 132: 1043-1054

14. Odom EB, Parikh RP, Um G, Kantola SW, Cyr AE, et al. (2018) NippleSparing Mastectomy Incisions for Cancer Extirpation Prospective Cohort Trial: Perfusion, Complications, and Patient Outcomes. Plast Reconstr Surg 142: 13-26.

15. Regnault P (1976) Breast ptosis: definition and treatment. Clin Plast Surg 3:193-203.

16. Moyer HR, Ghazi B, Daniel JR, Gasgarth R, Carlson GW (2012) Nipplesparing mastectomy: technical aspects and esthetic outcomes. Ann Plast Surg 68: 446-450.

17. Adam H, Bygdeson M, de Boniface J (2014) The oncological safety of nipple-sparing mastectomy - a Swedish matched cohort study.Eur J Surg Oncol 40: 1209-1215.

18. Simmons RM, Fish SK, Gayle L, La Trenta GS, Swistel A, et al. (1999) Local and distant recurrence rates in skin-sparing mastectomies compared with non-skin-sparing mastectomies. Ann Surg Oncol 6: 676-681.
19. Newman LA, Kuerer HM, Hunt KK, Kroll SS, Ames FC, et al. (1998) Presentation, treatment, and outcome of local recurrence after skin-sparing mastectomy and immediate breast reconstruction. Ann Surg Oncol 5: 620-626.

20. Davies K, Allan L, Roblin P, Ross D, Farhadi J (2011) Factors affecting post-operative complications following skin sparing mastectomy with immediate breast reconstruction. Breast 20: 21-25.

21. Munhoz AM, Aldrighi CM, Montag E, Arruda EG, Aldrighi JM, et al. (2013) Clinical outcomes following nipple-areola-sparing mastectomy with immediate implant-based breast reconstruction: a 12-year experience with an analysis of patient and breast-related factors for complications. Breast Cancer Res Treat 140: 545-555.

22. Folli S, Mingozzi M, Curcio A, Buggi F, Rossi C (2015) Nipple-Sparing Mastectomy: An Alternative Technique for Large Ptotic Breasts. J Am Coll Surg 220: e65-e69.

23. Tondu T, Hubens G, Tjalma WA, Thiessen FE, Vrints I, et al. (2020) Breast reconstruction after nipple-sparing mastectomy in the large and/or ptotic breast: A systematic review of indications, techniques, and outcomes. J Plast Reconstr Aesthet Surg 73: 469-485.

24. Hollander E (1924) Die operation dermamma hypertrophy underage brust. Deutsch Med Wochenschr 50: 1400.

25. Becker H, Lind JG $2^{\text {ss }}$, Hopkins EG (2015) Immediate Implant-based Prepectoral Breast Reconstruction Using a Vertical Incision. Plast Reconstr Surg Glob Open 3: e412.

26. Erol OO, Spira M (1980) A Mastopexy Technique for Mild to Moderate Ptosis. Plast Reconstr Sur 65: 603-609.

27. Van Deventer PV (2004) The blood supply to the nipple-areola complex of the human mammary gland. Aesthetic Plast Surg 28 393-398. 